\title{
Genomic characterization of a novel group A lamb rotavirus isolated in Zaragoza, Spain
}

\author{
Max Ciarlet - Christian Hoffmann - Eleonora Lorusso - Rafael Baselga • \\ Maria Assunta Cafiero · Krisztián Bányai · Jelle Matthijnssens • \\ Viviana Parreño · Simona de Grazia $\cdot$ Canio Buonavoglia $\cdot$ Vito Martella
}

Received: 28 April 2008/Accepted: 28 June 2008/Published online: 26 July 2008

(C) Springer Science+Business Media, LLC 2008

\begin{abstract}
An ovine rotavirus (OVR) strain, 762, was isolated from a 30-day-old lamb affected with severe gastroenteritis, in Zaragoza, Spain, and the VP4, VP7, VP6, NSP4, and NSP5/NSP6 genes were subsequently characterized molecularly. Strain OVR762 was classified as a $\mathrm{P}[14]$ rotavirus, as the VP4 and VP8* trypsin-cleavage product of the VP4 protein revealed the highest amino acid (aa) identity (94\% and $97 \%$, respectively) with that of the P11[14] human rotavirus (HRV) strain PA169, isolated in Italy. Analysis of the VP7 gene product revealed that OVR762 possessed G8 serotype specificity, a type common in ruminants, with the highest degree of aa identity
\end{abstract}

Nucleotide sequence accession numbers. Sequence data reported in this manuscript have been deposited in the GenBank Data Library under Accession Numbers EF554151 (VP4), EF554153 (VP7), EF554157 (NSP4), EF554152 (VP6), and EF554158 (NSP5/NSP6) for ovine rotavirus strain OVR762.

\section{Ciarlet $(\square)$}

Vaccine \& Biologics - Clinical Research, Merck Research

Laboratories, Mailstop UG3CD-28, P.O. Box 1000,

North Wales, PA 19454-1099, USA

e-mail: max_ciarlet@merck.com

C. Hoffmann

Department of Microbiology, University of Pennsylvania,

Philadelphia, PA, USA

E. Lorusso · C. Buonavoglia · V. Martella

Dipartimento di Sanità e Benessere Animale,

Facoltà di Medicina Veterinaria di Bari, Bari, Italy

R. Baselga

Exopol, Zaragoza, Spain

M. A. Cafiero

Istituto Zooprofilattico di Puglia e Basilicata,

Sezione di Foggia, Italy
(95-98\%) shared with serotype G8 HRV, bovine rotavirus, and guanaco (Lama guanicoe) rotavirus strains. Moreover, strain OVR762 displayed a bovine-like NSP4 (genotype E2) and NSP5/NSP6 (genotype H3), and a VP6 genotype $\mathrm{I} 2$, as well as a long electropherotype pattern. This is the first report of a lamb rotavirus with $\mathrm{P}[14]$ and G8 specificities, providing additional evidence for the wide genetic and antigenic diversity of group A rotaviruses.

Keywords Rotavirus · Gastroenteritis · Genome · Genotype - Genetic diversity - Reassortment - Lamb · VP4 $\cdot$ VP6 $\cdot$ VP7 $\cdot$ NSP4 $\cdot$ NSP5

Present Address:

K. Bányai

Veterinary Medical Research Institute, Hungarian Academy

of Sciences, Budapest, Hungary

J. Matthijnssens

Department of Microbiology \& Immunology, Rega Institute for Medical Research, University of Leuven, Leuven, Belgium

V. Parreño

Virology Institute, CICV y A, National Institute of Agriculture

Technology (INTA), Buenos Aires, Argentina

S. de Grazia

Dipartimento di Igiene e Microbiologia, Università di Palermo, Palermo, Italy 


\section{Introduction}

The rotavirus genus is divided into seven antigenically distinct groups or serogroups (A to G), but group A rotaviruses are the main cause of acute viral gastroenteritis in humans and animals throughout the world [1]. Sheep are one of the few species in which group B rotaviruses appear to be more prevalent in neonatal lambs than group $\mathrm{A}$ rotaviruses [2, 3]. The VP6 protein of group A rotaviruses bears the subgroup (SG) specificities that allow antigenic classification of these viruses into SG I, SG II, both SG I and II, or into neither SG based on reactivity with SG specific monoclonal antibodies [1]. The VP6 genogroup, predictive of the VP6 SG specificity, may be determined by sequence analysis of a 379 bp fragment, spanning from amino acid (aa) 241367 [4].

The two outer capsid proteins, VP4 and VP7, associated with $\mathrm{P}$ and $\mathrm{G}$ serotype specificity, respectively, independently elicit neutralizing antibodies, and induce protective immunity. So far, out of 19 different $\mathrm{G}$ genotypes and 27 different $\mathrm{P}$ genotypes (defined by sequence analysis and/or nucleic acid hybridization data and designated in brackets), only 14 P serotypes (P1A, P1B, and P2 to P13) and 14 G serotypes (G1 to G14) have been identified by serology $[1,5]$.

The non-structural glycoprotein NSP4, the viral enterotoxin, and recent sequence analyses have revealed that the NSP4 gene of group A rotaviruses may be genetically classified into 11 genotypes, E1 to E11 [5], which correspond to the five genotypes, KUN (A)-, Wa (B)-, Au-1 (C)-, EW (D)-, or avian (E)-like, described previously [6-9]. Within NSP4 genotypes E1 and E2, previously genotypes B and A, respectively, rotavirus strains generally cluster according to species of origin, suggesting a constant pattern of evolution within species [7]. Genome segment 11 encodes NSP5, a phosphoprotein with kinase activity, and, via an out-of-phase open reading frame (ORF), a smaller, likely dispensable protein, NSP6 [1]. The NSP5/ NSP6 gene has been shown to be suitable to trace species of origin [10] and can be genetically classified into six genotypes (H1 to $\mathrm{H} 6)$ [5].

Despite the importance of sheep as farming animals, information of group A ovine rotavirus (OVR) strains is scanty $[2,3,11,12]$. In the late 1970s, an OVR strain (K923), isolated from a diarrheic lamb, was used to study the pathogenicity of OVRs under experimental conditions using gnotobiotic lambs [12-15]. Subsequently, three additional OVR strains, L-1, L-2, and L-3, were isolated in Japan during an outbreak of diarrhea in a sheep farm $[16,17]$. The partially characterized OVR strain L-1 was shown to agglutinate erythrocytes and not antigenically related to G3, G4, G5, or G6 rotaviruses [16]. The most well-known OVR strain, Lp14, was isolated from a diarrheic lamb in China in 1981, and belongs to VP6 SGI, VP4 genotype P[15], VP7 serotype G10, and NSP4 genotype E2 (previously genotype A) [5, 18-20]. Strain Lp14 has recently been licensed for use as a human vaccine in China (Lanzhou Institute of Biological Products). Antigenic analyses of the original K923 strain [12] and three additional OVR strains, LRV1 (SGI), LRV2a (SGI), and LRV2c (SGII), isolated from lamb feces in the United Kingdom, revealed that the OVR strain K923 was SGI, $\mathrm{P}[15]$, and G10 like the strain Lp14, while each of the remaining strains possessed unique antigenic properties commonly found in either cows or humans: P6[1]G3 (LVR1), P8[11]G6 (LVR2a), and P1A[8]G9 (LRV2c) [3].

In recent years, epidemiological surveillance to monitor the appearance of novel rotavirus antigenic types has intensified throughout the world, yielding evidence for the increasing antigenic diversity of group A rotaviruses [21-23]. Although the number of characterized OVR strains is small, OVRs seem to represent a diverse group, suggesting that sheep may interchange rotaviruses readily. In the present study, we report the isolation and the molecular and genetic characterization of the VP4, VP7, VP6, NSP4, and NSP5/NSP6 of an OVR strain, 762, from a 30-day-old lamb affected with severe gastroenteritis, in Spain.

\section{Materials and methods}

\section{Virus isolation}

Strain OVR762 was isolated in 2002 in the province of Zaragoza, Spain, in a sheep farm from the feces of a 30 -week-old lamb, affected with severe diarrhea. An isolate was propagated onto African green monkey kidney (MA-104) cells as described [21-23]. Evidence of viral replication was checked both by the appearance cytopathic effect and indirect immunofluorescence test, using a rabbit hyperimmune serum raised against a group A rotavirus (strain Bo/A-125/96 P7[5],G6).

\section{Electropherotype (e-type) determination}

Viral dsRNA was extracted from infected cells showing $50 \%$ cytopathic effect. Cryolysates were extracted with Vertrel XF (Dupont, Wilmington, DE) as previously described [23] and clarified twice by centrifugation for $30 \mathrm{~min}$ at $4,800 \mathrm{~g}$. Subsequently, the supernatants were centrifuged for $8 \mathrm{~h}$ at $85,000 \mathrm{~g}$ on a $30 \%$ sucrose layer in SW28 rotor (Beckman Coulter, Inc., Fullerton, CA). After digestion of viral pellet with $1 \%$ sodium-dodecyl-sulfate (SDS) and proteinase $\mathrm{K} 1 \mu \mathrm{g} / \mathrm{ml}$ (Sigma-Aldrich, Milano, Italy), viral dsRNA was extracted and the e-type was visualized as described [21-23]. 


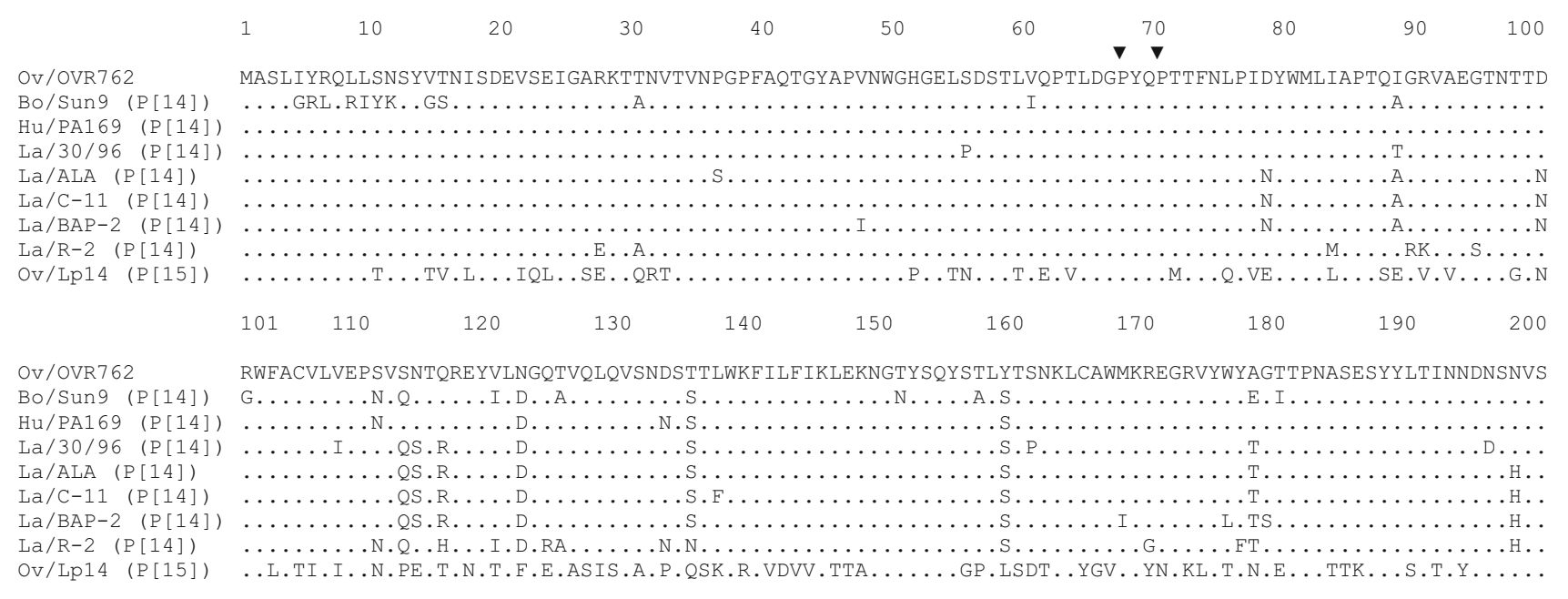

Ov/OVR762

$201 \quad 210 \quad * 220 \quad \underset{*}{*}{ }_{\text {CDAEFYLIPRSQTDLCAQYINNGLPPVQNTRNIVPVSISSREIRHTRAQMN }}^{230}$

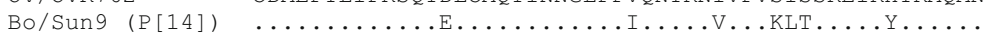

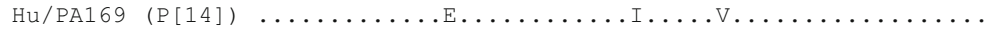

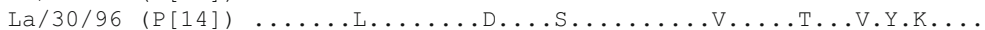

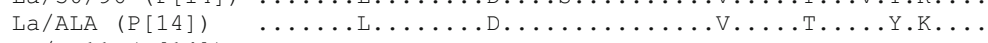

$\mathrm{La} / \mathrm{C}-11(\mathrm{P}[14]) \quad \ldots \ldots \mathrm{L} \ldots \ldots \ldots \mathrm{D} \ldots \ldots \ldots \ldots \ldots$. . . . . . . . . . . .

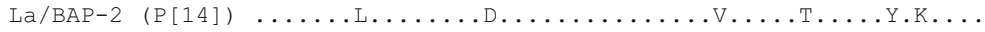

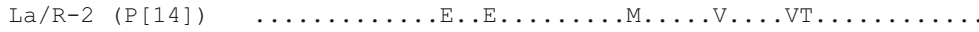

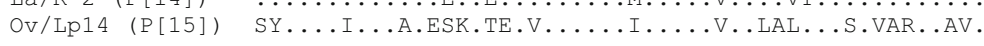

Fig. 1 Comparison of the deduced amino acid sequence of the outer capsid VP8* trypsin cleavage product of VP4 of the Spanish OVR762 strain with $\mathrm{P}$ [14] HRV, BRV, and lapine rotavirus strains, and P[15] Lp14 OVR strain. The highly conserved cysteine $(*)$, proline $(\boldsymbol{\nabla})$, and

RNA extraction and PCR amplification of the VP8*, VP7, VP6, NSP4, and NSP5/NSP6 genes

Viral dsRNA from the fecal specimen was extracted by adsorption on cellulose CF11 as described previously [23]. Viral dsRNA was denatured in dimethyl-sulfoxide (SigmaAldrich, Milano, Italy) at $97^{\circ} \mathrm{C}$ for $5 \mathrm{~min}$. Reverse transcription of dsRNA was carried out using MuLV-reverse transcriptase (Perkin-Elmer Europe B.V. Monza) while PCR amplification was carried out with AmpliTaq Gold ${ }^{\circledR}$ DNA polymerase (Perkin-Elmer Europe B.V. Monza).

The VP8* subunit of the VP4, the connecting peptide, and the N terminus of the VP5* subunit of VP4 (876 base pairs [bp]), predictive of the $\mathrm{P}$ genotype, were reverse transcribed and amplified as described [24, 25]. The fulllength sequence of VP4 (2,361 bp) was determined by using selected consensus $3^{\prime}$ end and internal primers as described elsewhere [24]. The full-length VP7 gene $(1,062 \mathrm{bp})$ was reverse transcribed and amplified using primer Beg9 [26] and the $3^{\prime}$ degenerated primer End9deg ( $5^{\prime}$-GGTCACATCDWMCARYTCTAAYYHM- $\left.{ }^{\prime}\right)$. The VP6 genogroup was determined by amplification of a 379bp fragment, spanning from amino acid (aa) 241-367 of the VP6, as described [4]. In addition, the complete VP6 gene segment $(1,355 \mathrm{bp})$ was amplified as described elsewhere [24]. The full-length gene (genome segment 10, $751 \mathrm{bp}$ ) arginine (ם) residues are indicated. The GenBank Accession Numbers of the VP8* sequences are listed in Table 1. Abbreviations: $\mathrm{Hu}$, human; La, lapine; Bo, bovine; Ov, ovine

encoding for the NSP4 protein and the NSP5/NSP6 fulllength gene (genome segment 11, $667 \mathrm{bp}$ ) were amplified as described [19, 27]. To obtain the complete nucleotide sequence of each segment analyzed in this study, the $5^{\prime}$ and $3^{\prime}$ terminal sequences were determined using a modified version of the single-primer amplification method, as described previously [5].

Sequence and phylogenetic analyses

For direct sequencing of PCR amplicons, three distinct amplicons were analyzed and a consensus sequence was determined. Sequences were assembled and analyzed using Bioedit software package (Department of Microbiology, North Carolina State University, USA) (http://www.mbio. ncsu.edu/BioEdit/bioedit.html). Sequence comparisons were also analyzed by BLAST searches [28].

Phylogenetic and molecular evolutionary analyses were conducted at the nucleotide level using MEGA version 2.1 (Arizona State University, USA) [29]. Phylogenetic trees based on the corresponding ORF for the VP4, VP7, VP6, NSP4, and NSP5/NSP6 nucleotide sequences were elaborated with the neighbor-joining method, supplying a statistical support with bootstrapping over 100 replicates, according to the recently established rotavirus classification scheme [5]. 
Nucleotide sequence accession numbers

GenBank accession numbers EF554151, EF554153, EF554152, EF554157, and EF554158 were assigned to VP4, VP7, VP6, NSP4, and NSP5, respectively, of OVR762 strain.

\section{Results}

Analysis of the electropherotype (e-type)

The e-type of OVR762 strain displayed a long electrophoretic pattern $4: 2: 3: 3$, which is typical of group A rotaviruses (data not shown).

Molecular characterization of the spike protein VP4 of the OVR762 strain

To determine the $\mathrm{P}$ genotype of OVR762, we first sequenced the VP4 trypsin-cleavage product, VP8*, and subsequently, the full-length sequence of VP4 was determined. The VP4 gene was found to contain 2,361 bp with an open reading frame encoding a protein with a predicted size of 776 aa, one aa more than VP4s of most HRVs [30]. As with most rotavirus strains, the potential trypsincleavage sites at residues 231, 241, and 247 [31] (Fig. 1) were conserved in strain OVR762. In addition, the highly conserved prolines at residues 68, 71, 225, 226, 235, 334, 390, 395, 435, 451, 455, 483, 524, 669, 716, 749, and 761 and the cysteines at residues 216, 318, 380, and 774 were maintained. The deduced aa sequences based on the VP8* sequence of OVR762 were compared with those of representative strains of all 27 defined $P$ genotypes (Table 1 and data not shown), and the VP8* of OVR762 revealed high aa identities [90\% (La/R-2) to 97\% (Hu/PA169)] with those of $\mathrm{P}[14]$ strains. With the remaining $\mathrm{P}$ genotypes, the aa identity of OVR762 VP8* ranged from 35\% (Bo/KK3, $\mathrm{P} 8[11])$ to $83 \%$ (Hu/K8, P3[9]). In addition, BLAST search (http://www.ncbi.nlm.nih.gov) confirmed the genetic relatedness of OVR762 with P[14] strains of the VP4 gene (85-92\%) and its deduced protein sequence (93-97\%) as well (data not shown). Since it has been established that rotavirus strains that exhibit a VP4 aa identity of approximately $\geq 89 \%$ belong to the same $\mathrm{P}$ genotype [32, 33], our results indicate that the Spanish OVR762 strain belongs to the $\mathrm{P}[14]$ genotype.

Molecular characterization of the glycoprotein VP7 of the OVR762 strain

The basic structure of the VP7 gene from the OVR762 strain was similar to that of other rotavirus strains in that it presented two in-phase open reading frames beginning at nucleotides 49-51 and 136-138, and a single TAG codon at nucleotides 1,027-1,029, coding for a VP7 of 297 or 326 amino acids, respectively. Two potential N-linked glycosylation sites located at aa 69 and 238 were found, as in all other serotype G8 HRV and bovine rotavirus (BRV) strains, with the exception of strains that lack the glycosylation site at position 69 (BRV 678) and at position 238 (guanaco rotavirus [GRV] strain Río Negro) [24, 34-43] (Fig. 2). The deduced aa sequence for the gene encoding the VP7 of OVR762 with those of representative rotavirus strains from other $\mathrm{G}$ types was also compared (Table 1 and data not shown). The highest degree of aa identity (95-98\%) of the VP7 of OVR762 was found with the serotype G8 HRV, BRV, and GRV strains. Among serotype G8 BRV strains, the VP7 of OVR762 was most closely related (96-97\%) to those of Japanese (Niigata, Sun9, BRV16), Scottish (678), and American (Cody I-801) strains. The aa identity with serotype G8 GRV strains, Chubut and Río Negro (isolated in Argentina), was 97\% and $98 \%$, respectively, while the aa identities to G8 HRV strains (isolated in different parts of the world) ranged from 95\% (South African GRV570) to 98\% (Finnish HAL1166). The aa sequence analysis of the VP7 glycoprotein revealed that the sequence similarity in the four variable regions, A (aa 87-101), B (aa 141-152), C (aa 208-224), and F (aa 235-242) [44], between OVR762 and BRV, HRV, or GRV G8 strains clearly supports the inclusion of OVR762 as serotype G8 (Fig. 2). With the remaining serotypes, aa identity ranged from $57 \%$ with avian rotavirus Ch-1 (G19) to $85 \%$ with the simian rotavirus RRV (G3).

\section{Molecular characterization of the inner capsid protein} VP6 of the OVR762 strain

Comparative analysis of the deduced aa sequences of the fragment of VP6 (aa 241-367) suggested that OVR762 belonged to the genotype $\mathrm{I} 2$ (data not shown). The deduced aa sequence of the complete VP6 protein exhibited a high aa $(99 \%)$ sequence identity to that of the bovine strain UK (SGI) (Table 1). The aa identity to HRV and BRV SGI strains, all of which also belong to the VP6 genotype I2, ranged from $92 \%$ to $99 \%$, while that to SGI porcine rotavirus strains showed a range of 90-91\% (Table 1 and data not shown). The residues 305 and 315 of the VP6 of OVR762 were Ala and Glu, respectively, a pattern that is consistent with the non-porcine SGI specificity. Although the VP6 of OVR762 belongs to genotype I2, the VP6 protein of OVR762 was $98 \%$ identical to that of the ovine strain Lp14, which belongs to closely related genotype I10 (Table 1) [5]. However, at the nucleotide level, the VP6 of OVR762 was only $84.7 \%$ identical to that of Lp14. Since VP6 is a rather conserved protein that exhibits little aa 


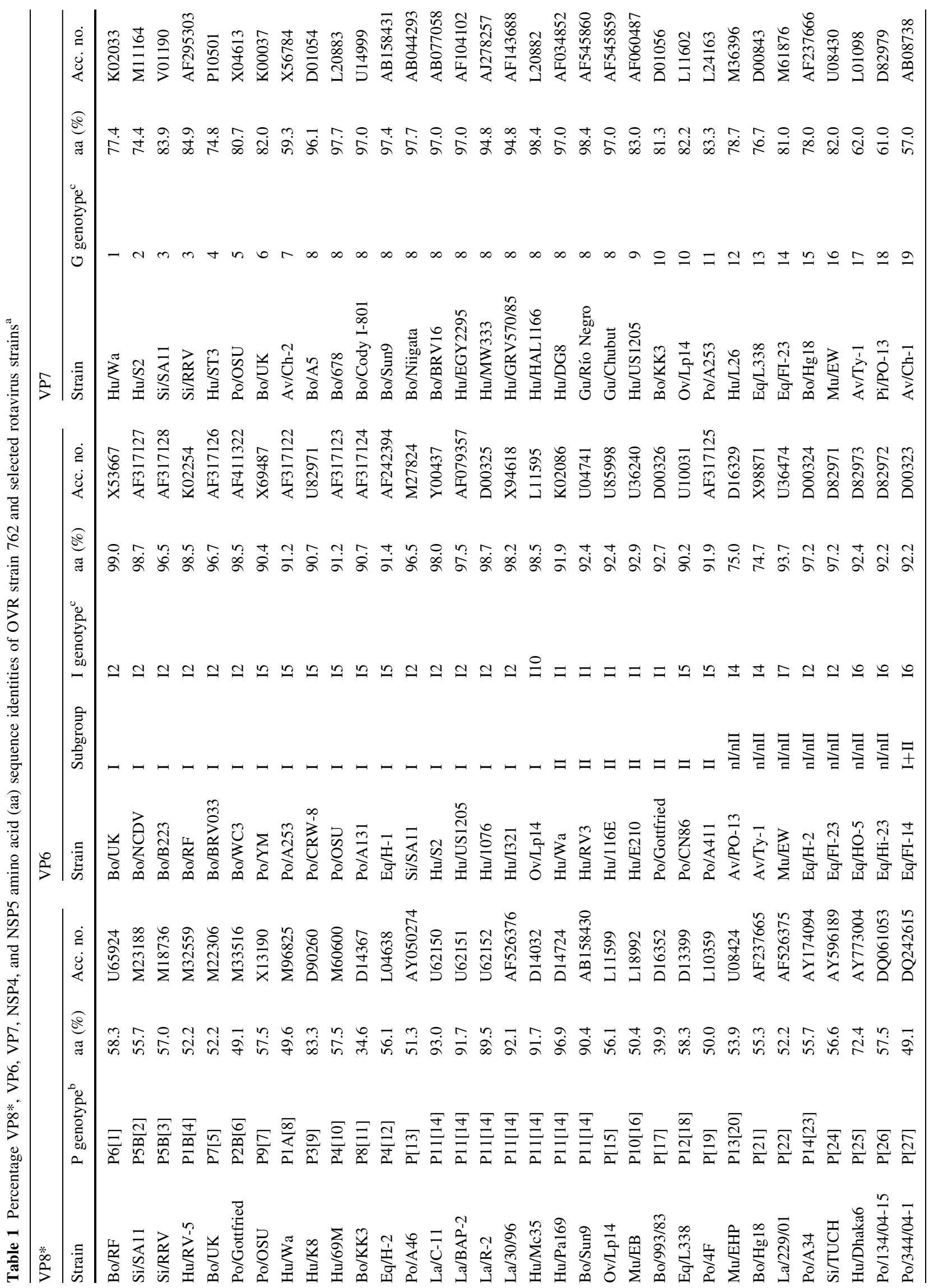




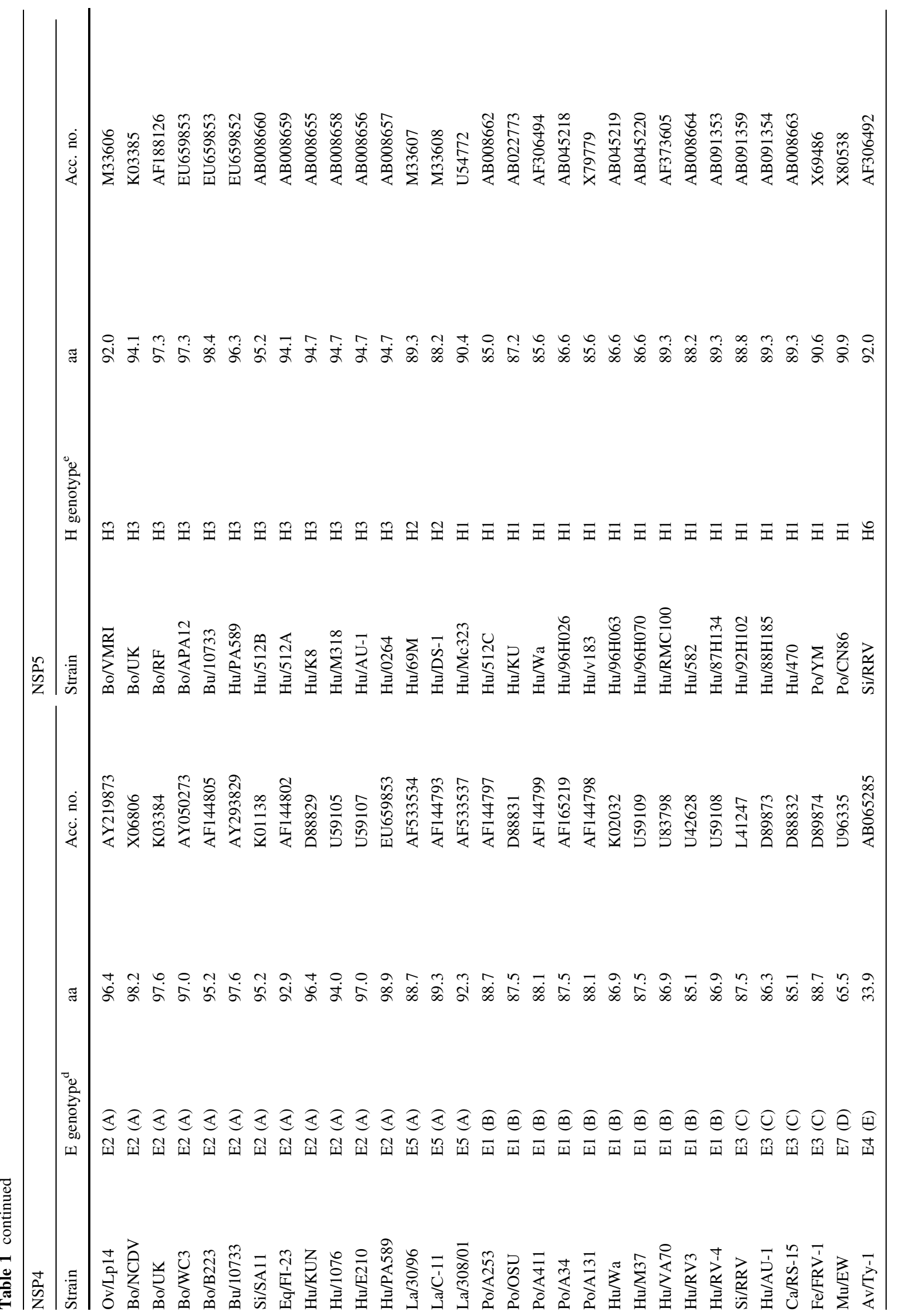




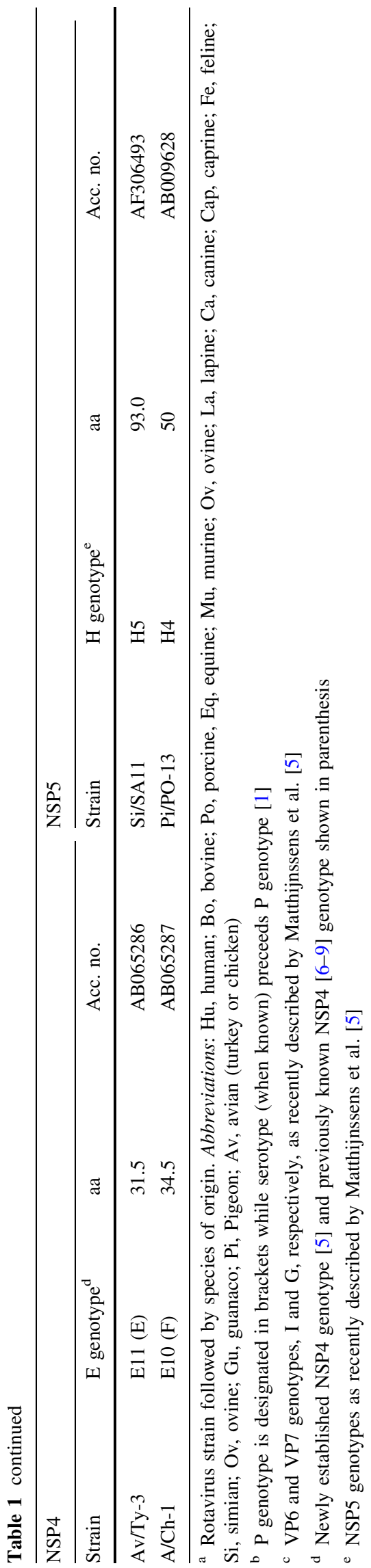

sequence diversity $[1,5]$, this finding strengthens the preference to use nucleotide sequences above amino acid sequences for genotyping purposes, as was suggested previously [5].

Molecular characterization of the viral enterotoxin NSP4 of the OVR762 strain

The fundamental structure of the NSP4 gene of OVR762 sequenced in this study was similar to those of other rotavirus strains sequenced previously, consisting of an open reading frame encoding a protein with a predicted size of 175 aa. The deduced aa sequence contained two potential $\mathrm{N}$-linked glycosylation sites located at aa 8 and 18, two cysteine residues at aa 63 and 71, and histidine-131 (a key residue for enterotoxigenic activity) which are conserved in NSP4 proteins [7, 10, 21] (Fig. 3). The deduced aa sequence of the NSP4 of OVR762 exhibited 93-99\% aa identity with ovine, bovine, simian, equine, and human rotavirus strains in the NSP4 genotype E2, previously knows as genotype A (Table 1, and data not shown) [5, 7, 10]. The NSP4 of OVR762 exhibited the greatest aa identity (99\%) with the genotype E2 HRV strain PA589, isolated in Italy, while the range of aa identities to other HRV strain belonging to the same genotype E2 was 94-97\%. The NSP4 of OVR762 exhibited an aa identity of $96 \%$ with that of the OVR strain Lp14, while the aa identity of the NSP4 of OVR762 to most BRV strains (all genotype E2) ranged from $95 \%$ to $98 \%$. The aa identities with rotavirus strains belonging to the remaining NSP4 main genotypes were 89-93\% (genotype E5, lapine strains previously included within genotype A), 85-89\% (genotype E1, previously known as genotype B), 85-89\% (genotype E3, previously known as genotype C), $66 \%$ (genotype E7, previously known as genotype D), and 31-35\% (genotypes E4, E10, or E11, previously genotypes $\mathrm{E}$ or F).

Molecular characterization of the NSP5/NSP6 of the OVR762 strain

The complete sequence of the NSP5/NSP6 genome segment 11 was 667 nucleotides (nt) long, with the sequence coding for NSP5 located between nt 22 and 618, and a second out-of-phase ORF (coding for NSP6) conserved between nt 80 and 358. The predicted NSP5 and NSP6 proteins of OVR762 were 198 and 92 aa in length, respectively (data not shown). The serine residues (153, 155,163 , and 165) involved with phosphorylation [10], the cysteine residues at position 171 and 174, and the highly conserved $\mathrm{COOH}$ terminus in the NSP5 protein were maintained. The deduced aa sequence of the NSP5/NSP6 of OVR762 was compared with NSP5 sequences of representative rotavirus strains (Table 1), and the aa identity 


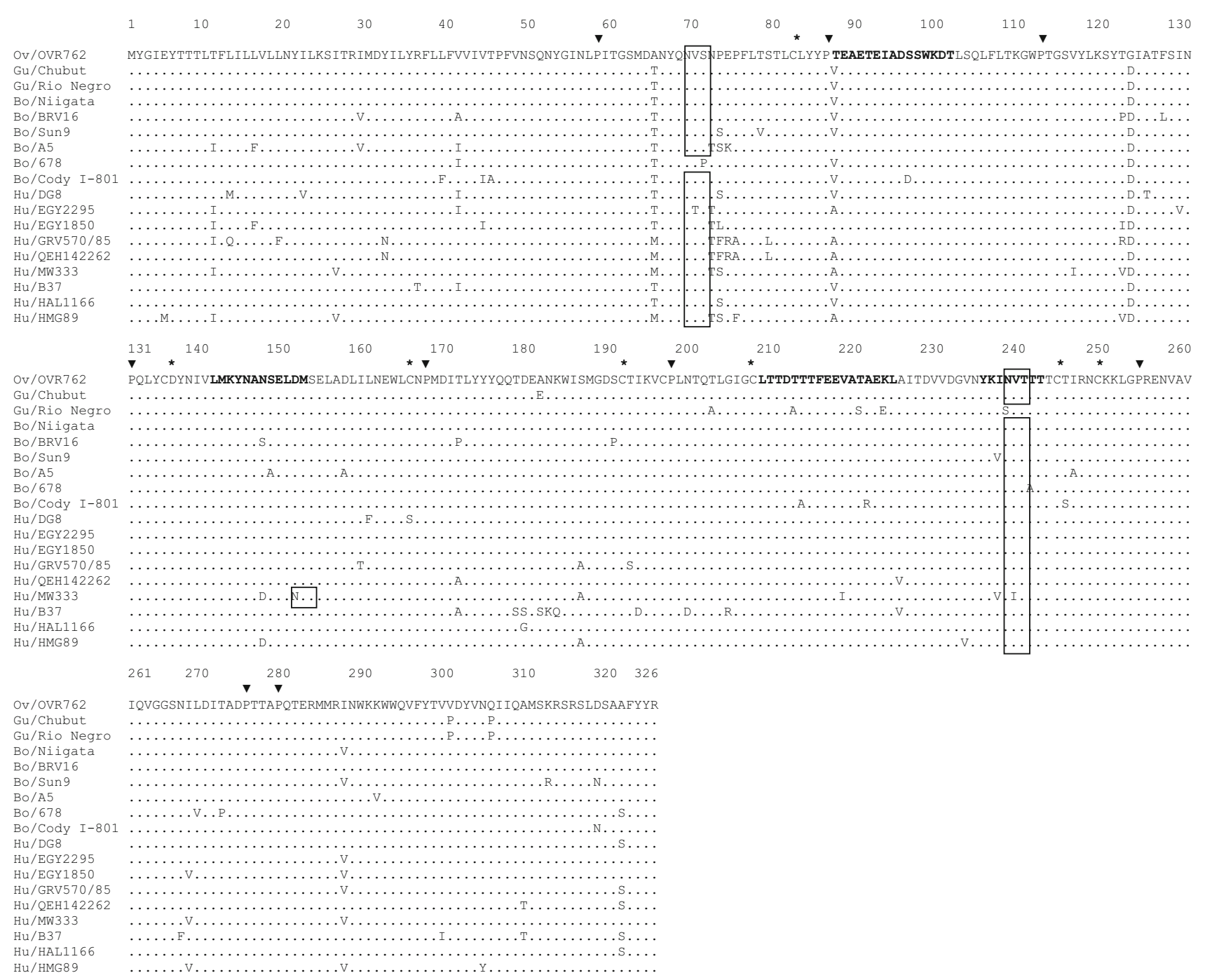

Fig. 2 Comparison of the deduced amino acid sequence of VP7 of strain OVR762 with serotype G8 BRV, HRV, and guanaco rotavirus strains. Variable regions A, B, C, and F (amino acids 87-101, 141152, 208-224, 235-242, respectively) [45] are shown in boldface type. Potential N-linked glycosylation sites are shown in boxes.

of the NSP5/NSP6 of OVR762 with those of representative rotavirus strains ranged from $98 \%$ (buffalo rotavirus strain 10733, belonging to genotype $\mathrm{H} 3$ ) to $50 \%$ (avian strain PO-13, belonging to genotype H4). A high aa identity (94-96\%) was also found to some HRV strains belonging to the genotype $\mathrm{H} 3$, and to genotype $\mathrm{H} 3 \mathrm{BRV}$ strains RF and APA12 and UK (97 and 94\%, respectively).

Phylogenetic analyses and genotyping of VP4, VP7, VP6, NSP4, and NSP5/NSP6 of OVR762 strain

Phylogenetic analysis of the entire VP4 nucleotide sequence of OVR762 strain revealed a close genetic relationship to the G6P11[14] HRV strains PA169, MG6, and Hun5, isolated in Italy, Australia, and Hungary,
Cysteine $(*)$ and proline $(\boldsymbol{\nabla})$ residues conserved in all strains are indicated because they are thought to be important in the conformation of the protein. The GenBank Accession Numbers of the VP7 sequences are listed in Table 1. Abbreviations: Hu, human; Bo, bovine; Gu, guanaco; Ov, ovine

respectively, and the G8P11[14] guanaco rotavirus strain Chubut, isolated in Argentina, as evidenced in Fig. 4a. The nucleotide identities between the VP4 gene segment of OVR762 and the other $\mathrm{P}[14]$ strains ranged from $80 \%$ to $97 \%$ (data not shown), supporting its classification as a $\mathrm{P}[14]$ strain. Among rotavirus strains with the serotype G8 specificity, the nucleotide sequence of the VP7 gene of the OVR762 clustered with G8 BRVs as well as rotavirus strains of camelid (Chubut and Río Negro) origin (Fig. 4b). The nucleotide identities between the VP7 ORF of OVR762 and the other G8 strains ranged from $82 \%$ to $95 \%$ (data not shown), confirming that OVR762 belongs to the G8 genotype. The phylogenetic tree of VP6 places OVR762 firmly within the I2 genotype, closely together with bovine, human, equine, and porcine rotavirus strains 


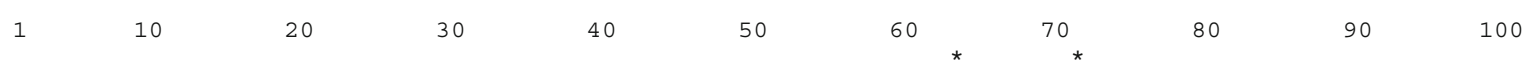

OV/OVR762 MEKLTDLNYTLSVITLMNSTRHTILEDPGMAYFPYIASVLTVLFTLHKASIPTMKIALKTSKCSYKVVKYCIVTIFNTLLKLAGYKEQITTKDEIEKQMD Ov/Lp14 $\mathrm{Bu} / 10733$ Bo/B223 $\mathrm{BO} / \mathrm{NCDV}$ $\mathrm{BO} / \mathrm{BRV} 033$ $\mathrm{BO} / \mathrm{WC} 3$ $\mathrm{BO} / \mathrm{UK}$ $\mathrm{Hu} / \mathrm{PA} 5$ / 89 $\mathrm{Hu} / \mathrm{A} 28$ $\mathrm{Hu} / \mathrm{KUN}$ $\mathrm{Hu} / \mathrm{S} 2$ $\mathrm{Hu} / \mathrm{E} 210$ $\mathrm{Hu} / \mathrm{RV} 5$ $\mathrm{Hu} / 1076$ La/ALA $\mathrm{La} / \mathrm{BAP}-2$ La/C-11 $\mathrm{La} / 30 / 96$ $\mathrm{La} / 229 / 01$ $\mathrm{La} / 308 / 01$ $\mathrm{La} / 160 / 01$ $\mathrm{La} / \mathrm{R}-2$ $\mathrm{Eq} / \mathrm{H}-2$ Eq/FI-23 Eq/FI- 14 Eq/HI-23

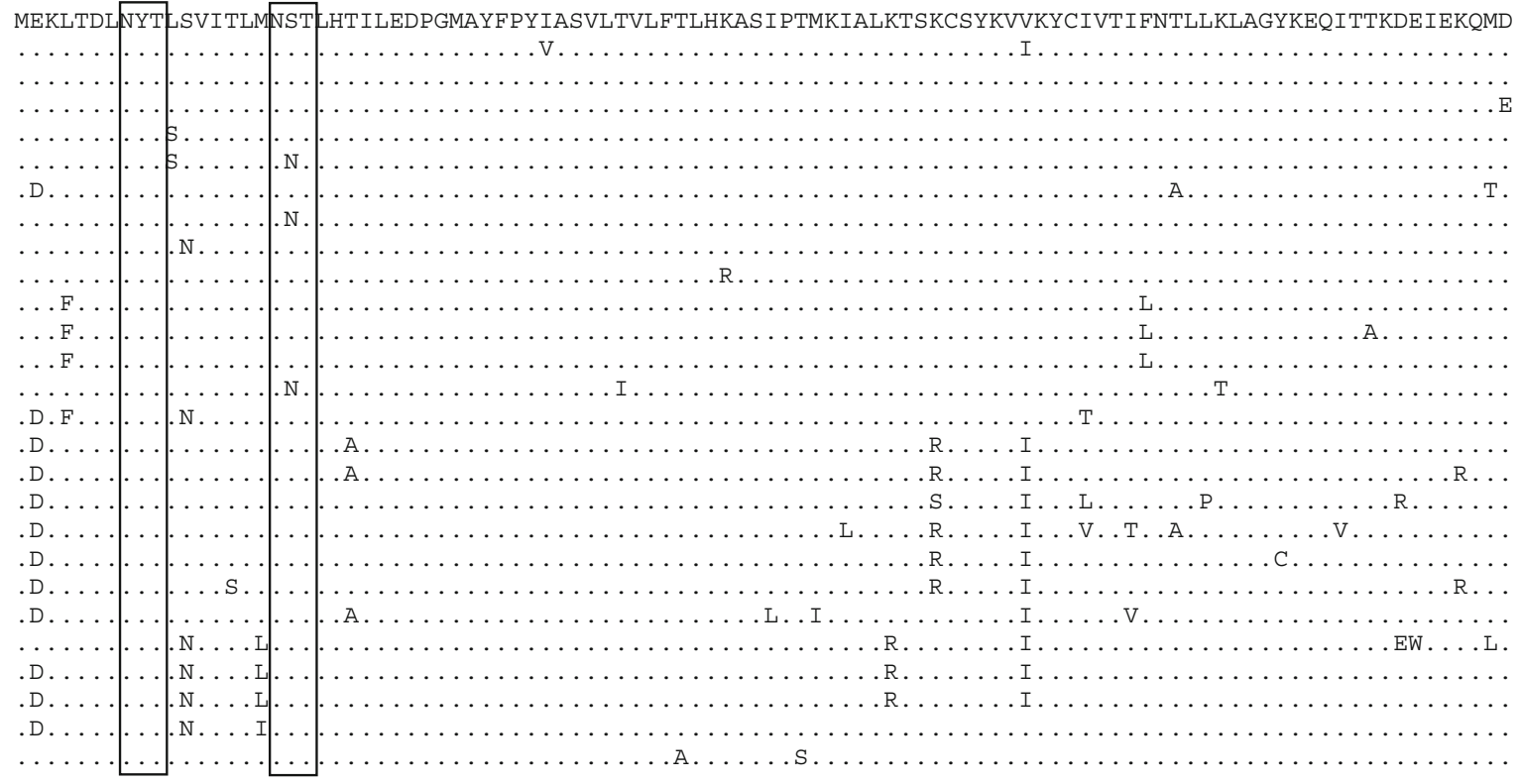

VP4-Binding Region

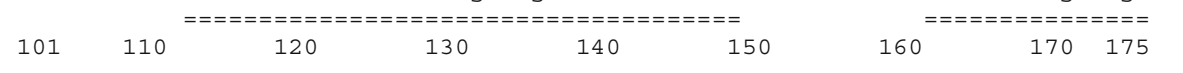

OV/OVR762 RVVKEMRRQLEMIDKLTTREIEQVELLKRIHDKLMVRPTDEIDMTKEINQKNVKTLEEWENGKNPYEPKEVTATM

Ov/Lp14

$\mathrm{Bu} / 10733$

Bo/B223

$\mathrm{BO} / \mathrm{NCDV}$

$\mathrm{BO} / \mathrm{BRV} 033$

$\mathrm{BO} / \mathrm{WC} 3$

$\mathrm{BO} / \mathrm{UK}$

$\mathrm{Hu} / \mathrm{PA} 5 / 89$

$\mathrm{Hu} / \mathrm{A} 28$

$\mathrm{Hu} / \mathrm{KUN}$

$\mathrm{Hu} / \mathrm{S} 2$

$\mathrm{Hu} / \mathrm{E} 210$

$\mathrm{Hu} / \mathrm{RV} 5$

Hu/1076

La/ALA

La/BAP - 2

La/C-11

$\mathrm{La} / 30 / 96$

$\mathrm{La} / 229 / 01$

$\mathrm{La} / 308 / 01$

$\mathrm{La} / 160 / 01$

$\mathrm{La} / \mathrm{R}-2$

$\mathrm{Eq} / \mathrm{H}-2$

Eq/FI-23

Eq/FI- 14

Eq/HI -23

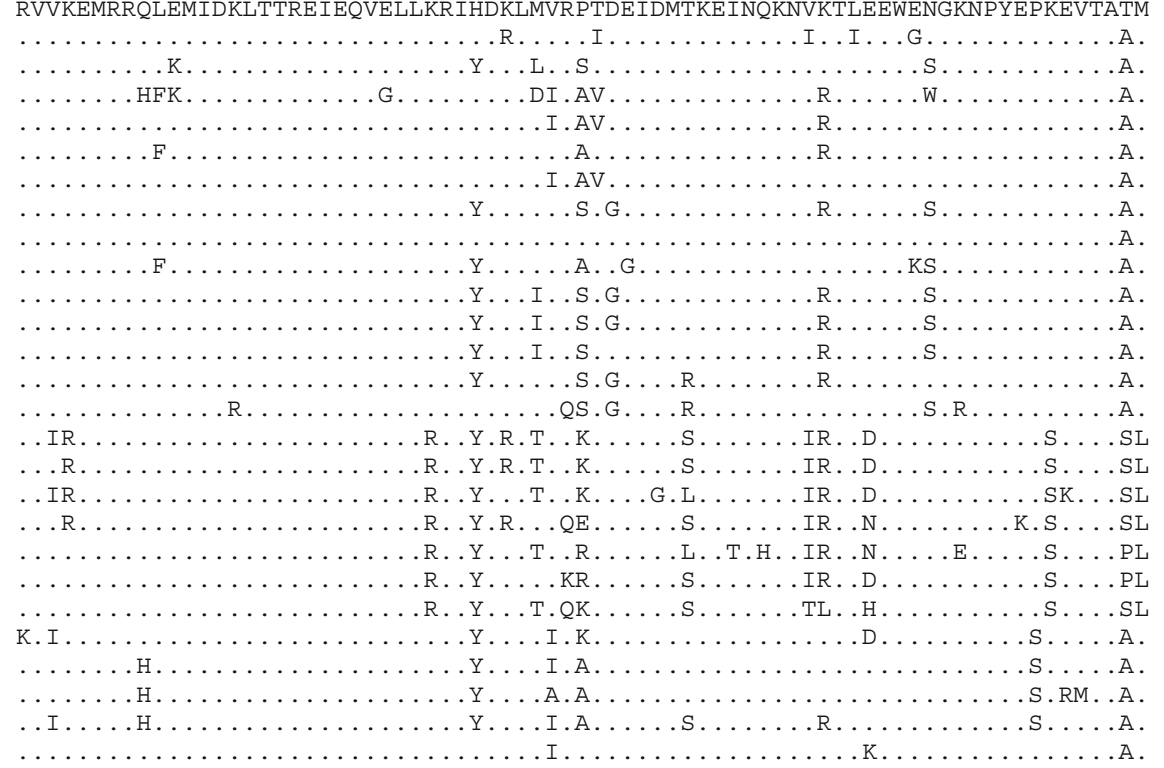

Fig. 3 Comparison of the deduced amino acid sequence of the NSP4 of strain OVR762 with NSP4 amino acid sequences of NSP4 genogroup A OVR, BRV, HRV, equine, and lapine rotavirus strains. Potential N-linked glycosylation sites are shown boxed and the two conserved cysteines are indicated (*). The VP4- and double-layered

(Fig. 4c), with identities between the VP6 gene segment and those of other I2 strains ranging from $84 \%$ to $95 \%$ (data not shown). Phylogenetically, the NSP4 sequence of OVR762 clustered very close with those of the human Hun5 strain and a number of other HRV strains that belong to the genotype E2 (Fig. 4d). A range of nucleotide identity (80-97\%) was found between the NSP4 gene segment of particle (DLP)-binding regions are indicated. The GenBank Accession Numbers of the NSP4 sequences are listed in Table 1. Abbreviations: $\mathrm{Hu}$, human; $\mathrm{Bu}$, buffalo; $\mathrm{Bo}$, bovine; Eq; equine; $\mathrm{La}$, lapine; Ov, ovine

OVR762 and those of other members of the E2 genotype (data not shown), confirming the classification of OVR762 as genotype E2. The NSP5/NSP6 phylogenetic tree (Fig. 4e) clustered OVR762 closely with HRV strains PA169 and BRV strains belonging to the NSP5/NSP6 genotype H3. The nucleotide identities of the NSP5/NSP6 gene of OVR762 and those of other members of the H3 
Fig. 4 Phylogenetic tree showing the relationships between the (a) VP4, (b) VP7, (c) VP6, (d) NSP4, and (e) NSP5/NSP6 nucleotide sequences of strain OVR762 (indicated in all phylogenetic trees with an arrow) with other rotavirus strains. The bootstrap values shown are percentages of 100 replications of the original data set. Abbreviations: $\mathrm{Hu}$, human; Bu, buffalo; Bo, bovine; Eq; equine; La, lapine; Ov, ovine; $\mathrm{Mu}$, murine; Cap, caprine; $\mathrm{Po}$, porcine; $\mathrm{Ca}$, canine; $\mathrm{Fe}$, feline; $\mathrm{Si}$, simian; $\mathrm{Gu}$, guanaco; $\mathrm{Pi}$, pigeon; Av, avian (turkey or chicken). All sequences were retrieved from GenBank and can be obtained upon request

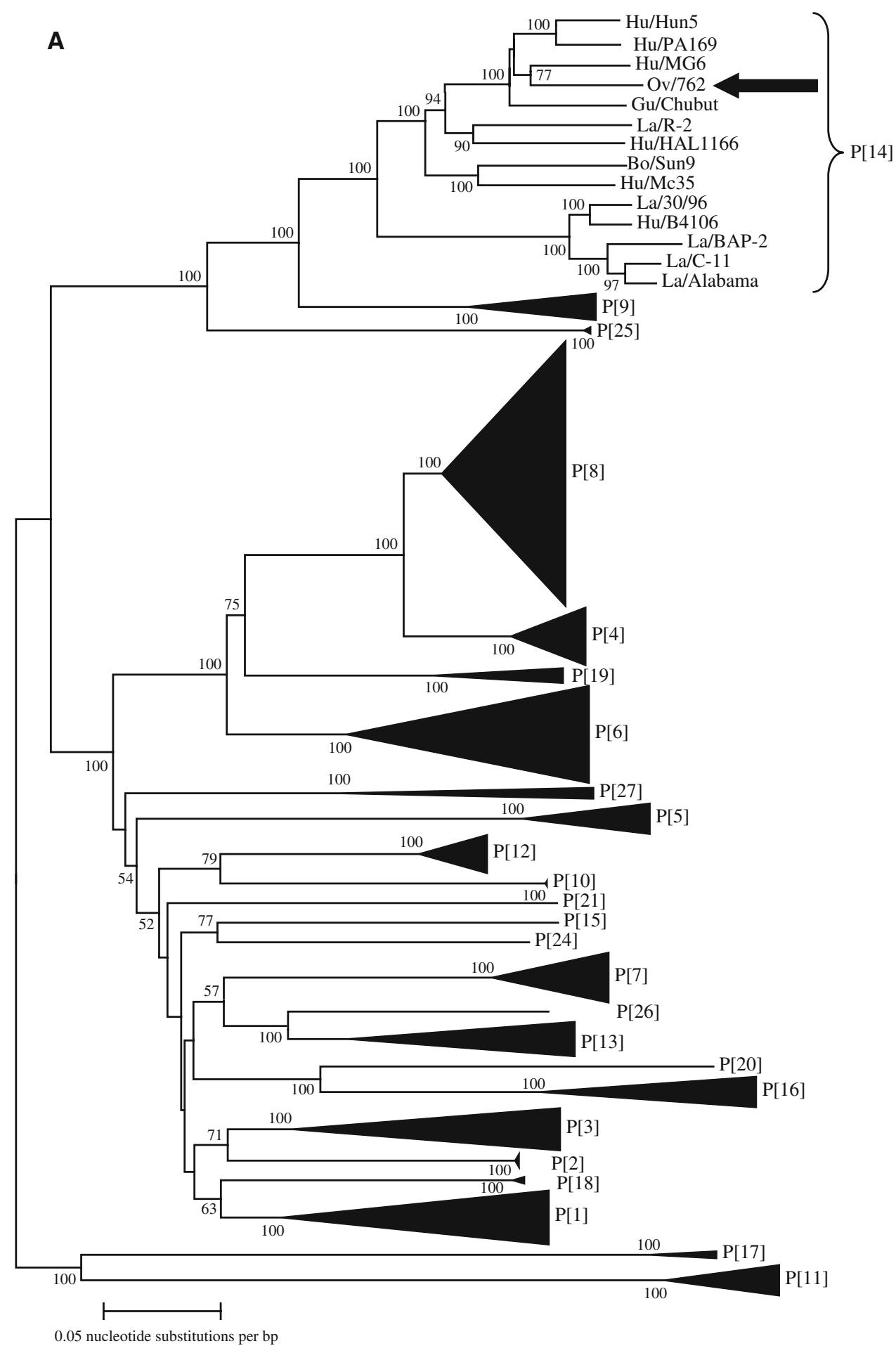

genotype ranged from $89 \%$ to $99 \%$ (data not shown), confirming the H3 genotype of the OVR762 strain.

\section{Discussion}

Although group A lamb rotaviruses were first identified in the 1970s [3, 11-13, 16-18] and a lamb rotavirus vaccine, containing OVR strain Lp14 (also known as Lanzhou Lamb Rotavirus [LLR]), is licensed for human use in China [45], the genotypic nature of OVRs circulating in diarrheic lambs is scanty because only a few OVR strains have been isolated and characterized to date. OVR strains have been isolated in the United Kingdom, Japan, and China, but only those isolated from the United Kingdom and China have been characterized $[3,13,16-20]$. Indeed, the most well- 
B

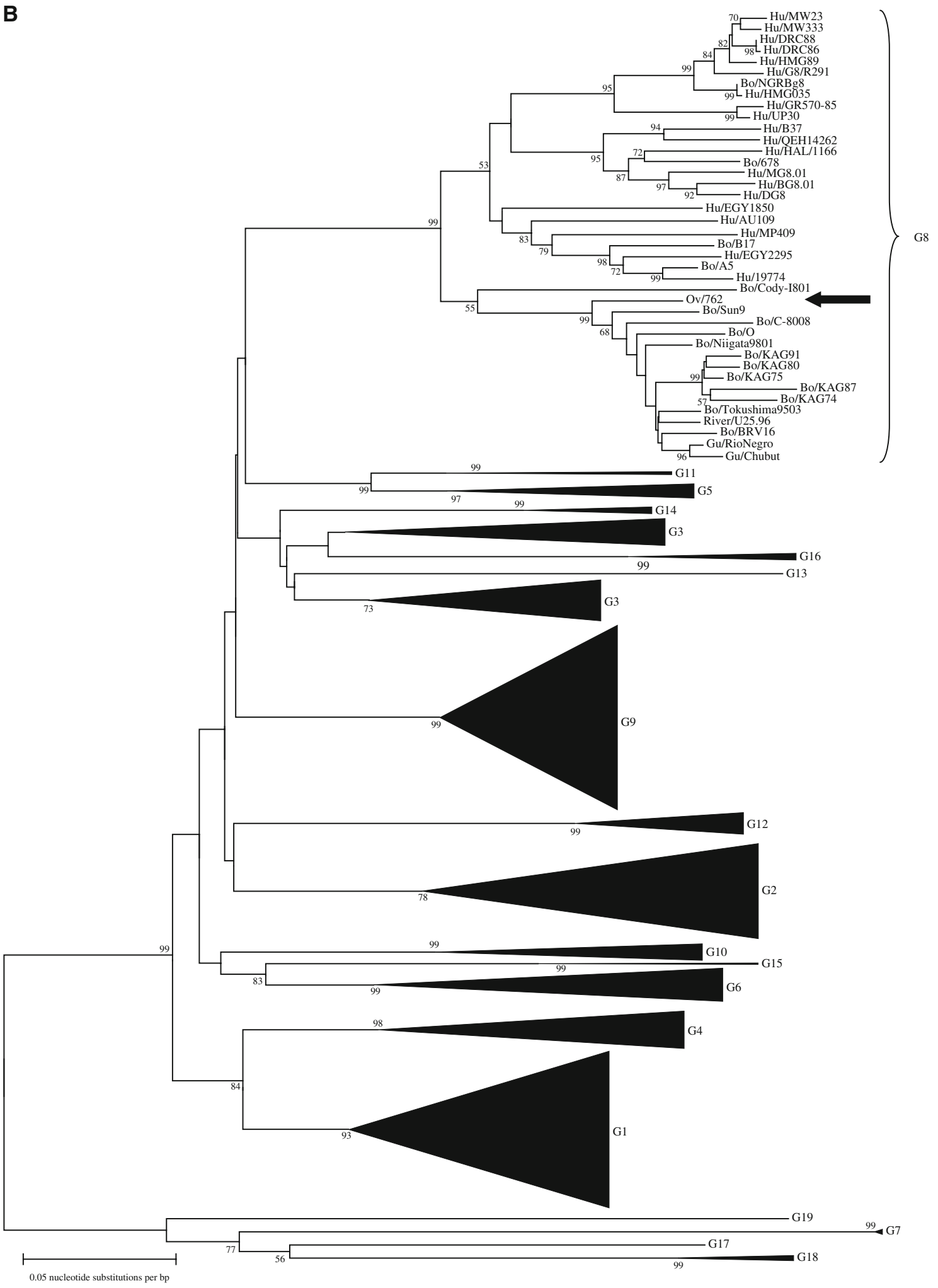

Fig. 4 continued

characterized OVR strains are the UK strain K293 and the Chinese virus Lp14, both of which display P[15] and G10 specificities [3, 13, 18]. Three additional OVR strains isolated in the United Kingdom were described to possess P6[1]G3 (LVR1), P8[11]G6 (LVR2a), and P1A[8]G9 (LVR2c) specificities [3], indicating a wide genetic 


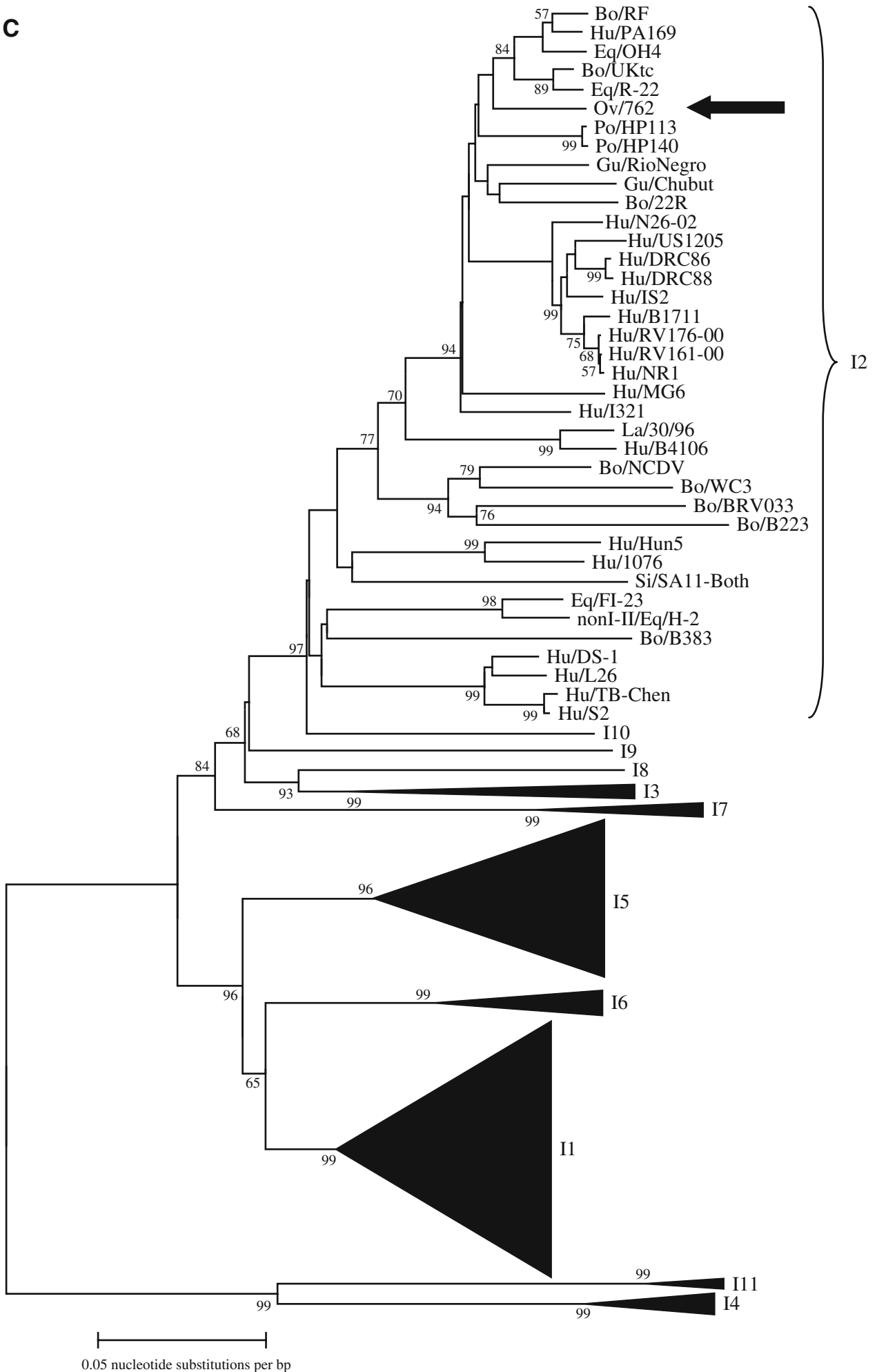

Fig. 4 continued

diversity among OVRs. However, the epidemiology of OVRs is still largely unknown, possibly because of lack of surveillance.
The present study constitutes the first report of an OVR strain, 762, with $\mathrm{P}[14]$ and G8 specificities. These data provide evidence for the wide genetic diversity of group A 
D

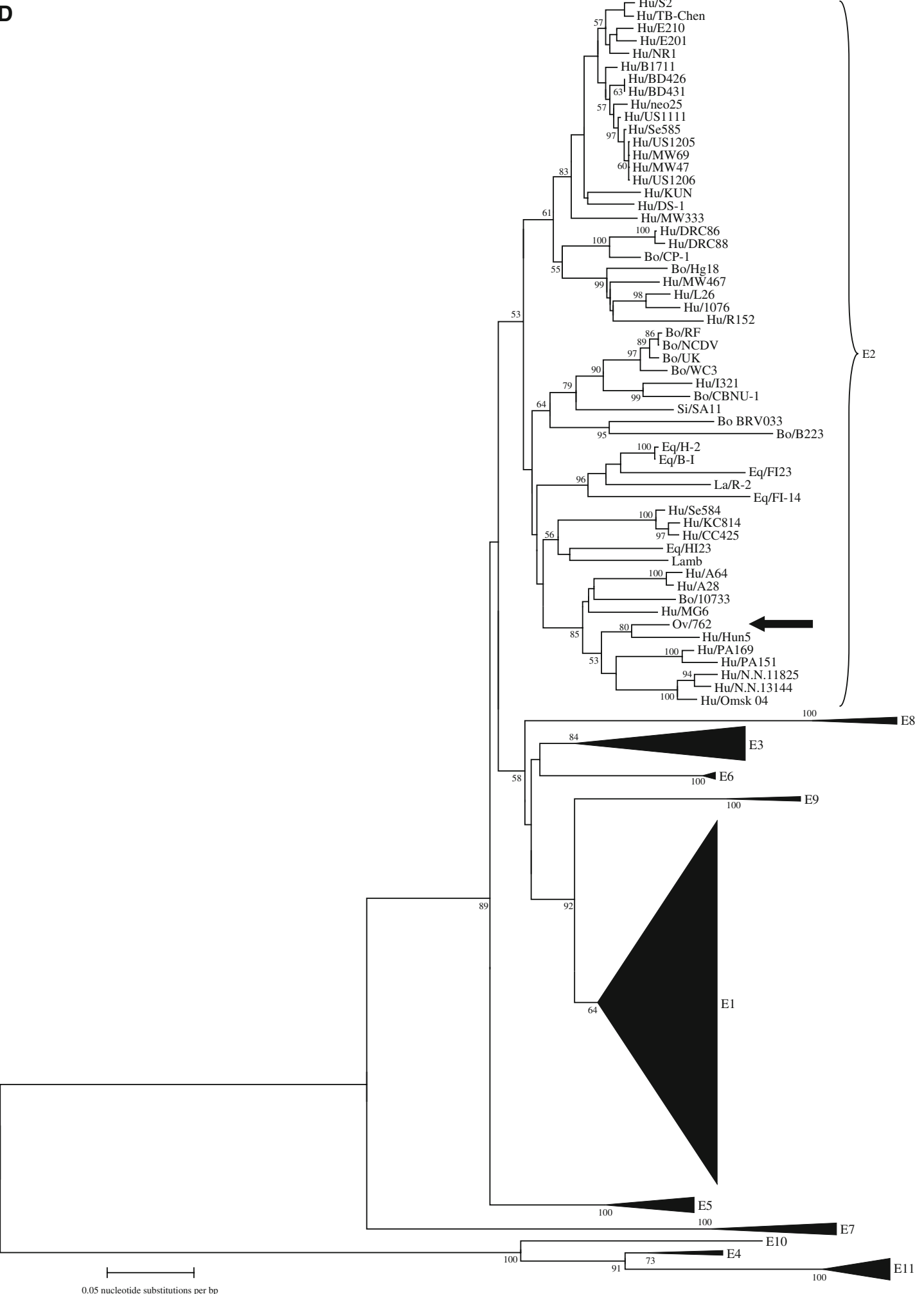

Fig. 4 continued

rotaviruses, and although the number of characterized OVR strains currently amounts to only six, OVRs seem to form a highly diverse group with broad genetic heterogeneity, as nearly all (5 out of 6) OVR isolates possess distinct $\mathrm{P}$ and $\mathrm{G}$ genotype combinations. Within the limits of the small number of OVR isolates available, and the fact that each of 


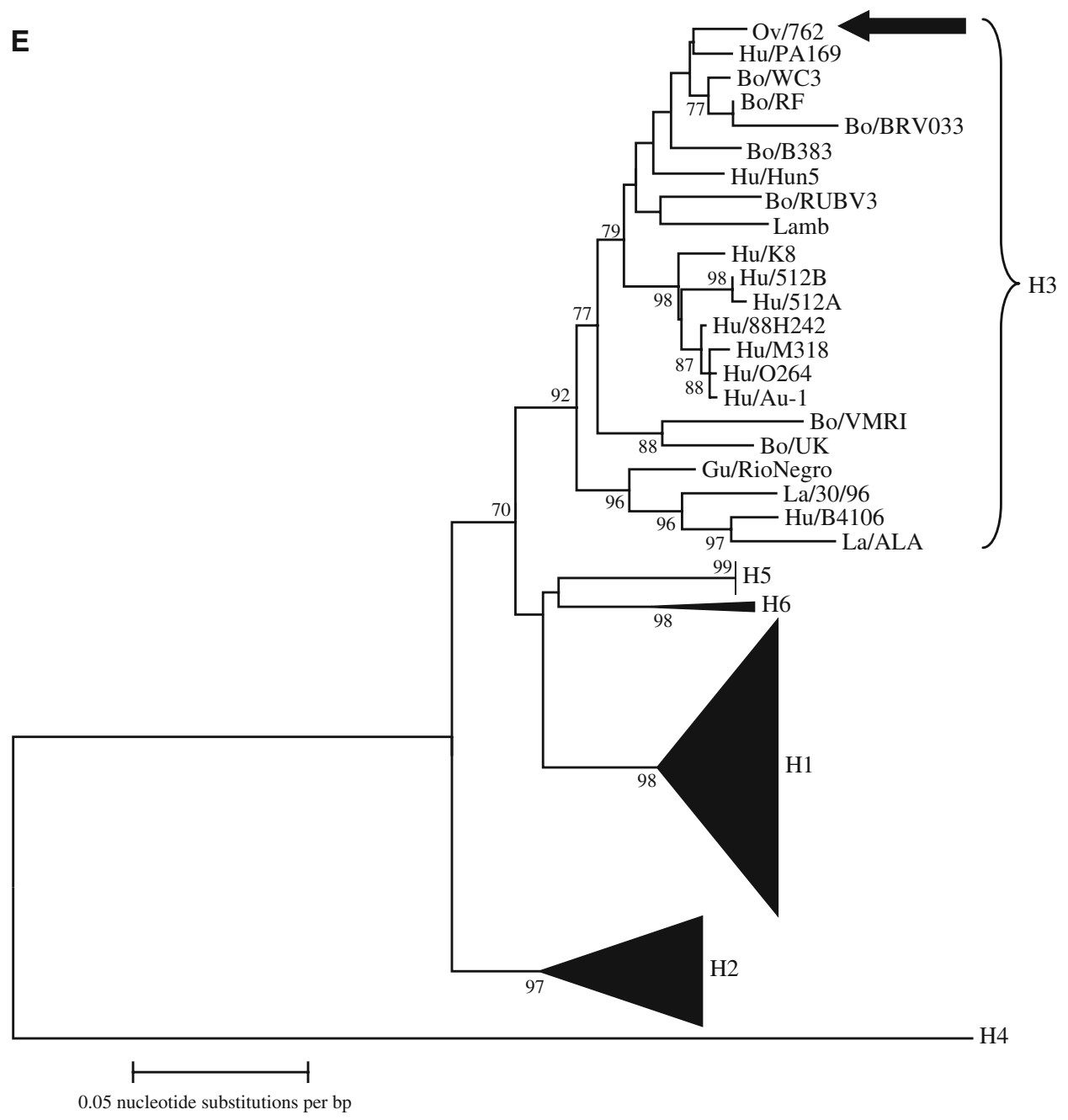

Fig. 4 continued

the 4 strains (K293, LVR1, LRV2a, LVR2c), isolated in the United Kingdom, and now OVR762, isolated in nearby Spain, are distinct OVR strains, it is tempting to speculate that there is no obvious tendency toward greater similarity among OVR strain being associated with a common geographical region. Although this could just be a sampling artifact, it is suggestive of a rapid and wide genetic diversification, which is not surprising, given the natural history of rotavirus infection in ruminants [2]. These data contrast with those of other animal rotaviruses, such as those of equine, bovine, and lapine origin, where certain $\mathrm{P}$ and $\mathrm{G}$ genotypes may predominate within a specific geographical locale or period of time [21, 30, 46-49].

Rotaviruses of serotype G8 specificity were first described in human in Indonesia [50] and subsequently in cattle [46]. Since their discovery, serotype G8 (in association with serotypes/genotypes P1A[8], P1B[4], P2A[6], $\mathrm{P} 4[10]$, $\mathrm{P} 6[1]$, or $\mathrm{P} 11[14])$ rotavirus strains have been isolated sporadically from children in several countries and have been established, after serotypes G6 and G10, as the third most common $G$ type (in association with serotypes/genotypes P6[1], P7[5], P8[11], or P11[14]) found in cattle [24, 34-43, 51-53]. In other animal species, a single equine rotavirus strain, 26/94, possessing P6[1]G8 specificities has been identified [49], as well as a single simian rotavirus strain, YK-1, with the same specificities [54]. Recently, two rotavirus strains, Chubut and Río Negro, isolated from guanacos (Lama guanicoe) in Patagonia (Argentina) exhibited P6[1]G8 or P11[14]G8 characteristics [37]. The phylogenetic analysis based on VP7 gene of OVR762 and G8 rotaviruses did not identify an apparent linkage with the VP4 specificity since different branches containing HRV and BRV strains, within the G8 rotaviruses, contained at least a P11[14] rotavirus strain, suggesting the occurrence of repeated interspecies transmissions and genetic reassortment events between ruminant and HRV strains.

The detection of the rotavirus P11[14] VP4 specificity, in association with serotype G8, in sheep raises additional questions on the origin and possible sources of introduction 
of this VP4 type in the genetic constellation of ruminants. After the original identification of the P11[14] VP4 type in HRV strains isolated in Italy and Finland [55], characterization of lapine rotavirus has also revealed that $\mathrm{P} 11[14] \mathrm{G} 3$ are a common $\mathrm{P} / \mathrm{G}$ combination in rotaviruses of rabbits $[21,30,56]$. Although the VP4 of the P11[14] lapine and HRV strains may have a common origin, it was always speculated that the HRV P11[14] strains are naturally occurring reassortants between humans and cattle [30]. Given that rotavirus strains with P11[14] VP4 specificity have been identified in a wide range of ruminants (cows, goats, guanacos, and now sheep) and humans in association with serotype G6 or G8 isolated in different parts of the globe, it might suggest the emergence of new rotavirus strains, via interspecies transmission, through contact of human and domestic ruminants. However, guanacos (Lama guanicoe), unlike alpacas (Lama alpaca) and llamas (Lama glama), are wild animals with considerable reduced contact with humans and domestic cattle raising the possibility that guanacos are one of the natural reservoir for P11[14]G8 rotavirus strains [37]. Increasing number of P11[14]G8 isolates makes, however, unlikely that they are the single reservoir as distinct lineages of the P11[14] VP4 gene has been detected in a variety of host species in various geographic locations (e.g., the Japanese G8 BRV strain, Sun9, and the European and African G8 HRV isolates, HAL1166 and EGY1850, respectively). It is also interesting that the OVR762 strain, isolated from a lamb in Spain, shares a close relatedness to a GRV strain, Chubut (P11[14]G8), from the Patagonia, Argentina. Guanacos are autochthonous from the Patagonia and have been in contact with sheep since they were introduced by the Spanish conquistadors. It would be of interest to elucidate the identity of the remaining rotavirus genes (VP1 to VP3 and NSP1 to NSP3) of the OVR strain 762 and compare it to the constellation of genes of not only the G8 or G6 P11[14] HRV and GRV strains, but also to that of the P11[14]G8 GRV strain Chubut.

This is the first time an OVR strain is reported from the Mediterranean area and the first time a P11[14]G8 OVR is described, and our data recognizes the complicated and diverse genetic divergence of OVRs. Regulatory authorities repeatedly have questioned whether introduction of a rotavirus vaccine would result in unusual rotavirus strains because of reassortment between vaccine strains and those in the environment. This study provides more evidence that these reassortments between rotaviruses primarily circulating in different species occur and will continue to occur with or without the introduction of a new rotavirus vaccine.

Acknowledgments The work was supported by grants from the University of Bari "Studio di Rotavirus Atipici Umani ed Animali".
J.M. was supported by the Institute for the Promotion of Innovation through Science and Technology in Flanders (IWT Vlaanderen).

\section{References}

1. M. Ciarlet, M.K. Estes, in Encyclopedia of Environmental Microbiology, ed. by G. Bitton (Wiley, New York, 2002), pp. 2753-2773

2. L.J. Saif, B. Rosen, A. Parwani, in Viral Infections of the Gastrointestinal Tract, 2nd edn., ed. by A.Z. Kapikian (Marcel Dekker, Inc., New York, 1994), pp. 279-367

3. T.A. Fitzgerald, M. Muñoz, A. Wood, D.R. Snodgrass, Arch. Virol. 140, 1541-1548 (1995). doi:10.1007/BF01322528

4. M. Iturriza-Gómara, C. Wong, S. Blome, U. Desselberger, J. Virol. Methods 105, 99-103 (2002). doi:10.1016/S0166-0934(02)00087-3

5. J. Matthijnssens, M. Ciarlet, E. Heiman, I. Arijs, T. Delbeke, S. Mc Donald et al., J. Virol. 82, 3204-3219 (2008). doi:10.1128/ JVI.02257-07

6. Y. Horie, O. Masamune, O. Nakagomi, J. Gen. Virol. 78, 23412346 (1997)

7. M. Ciarlet, F. Liprandi, M.E. Conner, M.K. Estes, Arch. Virol. 145, 371-383 (2000). doi:10.1007/s007050050029

8. H. Ito, M. Sugiyama, K. Masubuchi, Y. Mori, N. Minamoto, Virus Res. 75, 123-138 (2001). doi:10.1016/S0168-1702(01)00234-9

9. Y. Mori, M. Borgan, N. Ito, M. Sugiyama, N. Minamoto, J. Virol. 76, 5829-5834 (2002). doi:10.1128/JVI.76.11.5829-5834.2002

10. V. Martella, M. Ciarlet, A. Pratelli, S. Arista, V. Terio, G. Elia et al., J. Clin. Microbiol. 41, 5665-5675 (2003). doi:10.1128/ JCM.41.12.5665-5675.2003

11. M. Muñoz, M. Alvarez, I. Lanza, P. Carmenes, Epidemiol. Infect. 117, 203-211 (1996)

12. S. Wani, M. Bhat, R. Nawchoo, Z. Munshi, A. Bach, Trop. Anim. Health Prod. 36, 27-32 (2004). doi:10.1023/B:TROP.0000009523. 32254.45

13. D.R. Snodgrass, J. Herring, E. Gray, J. Comp. Pathol. 86, 637642 (1976). doi:10.1016/0021-9975(76)90073-6

14. D.R. Snodgrass, W. Smith, E. Gray, J. Herring, Res. Vet. Sci. 20, 113-114 (1976)

15. D.R. Snodgrass, A. Ferguson, F. Allan, K. Angus, B. Mitchell, Gastroenterology 76, 477-481 (1979)

16. T. Makabe, M. Sato, S. Ouneda, Y. Inaba, Arch. Virol. 90, 153-158 (1986). doi:10.1007/BF01314153

17. T. Makabe, H. Komaniwa, Y. Kishi, K. Yataya, H. Imagawa, K. Sato et al., Arch. Virol. 83, 123-127 (1985). doi:10.1007/BF01310971

18. S. Shen, B. Burke, U. Desselberger, Virology 197, 497-500 (1993). doi:10.1006/viro.1993.1620

19. K.V. Mohan, S. Kulkarni, R. Glass, B. Zhisheng, C.D. Atreya, Virus Genes 26, 185-192 (2003). doi:10.1023/A:1023491514820

20. K.V. Mohan, R. Glass, C.D. Atreya, Biologicals 34, 265-272 (2006). doi:10.1016/j.biologicals.2005.11.005

21. V. Martella, M. Ciarlet, A. Camarda, A. Pratelli, M. Tempesta, G. Greco et al., Virology 314, 358-370 (2003). doi:10.1016/S00426822(03)00418-5

22. V. Martella, M. Ciarlet, K. Bányai, E. Lorusso, S. Arista, A. Lavazza, G. Pezzotti, N. Decaro, A. Cavalli, M. Lucente, M. Corrente, G. Elia, M. Camero, M. Tempesta, C. Buonavoglia, J. Clin. Microbiol. 45, 577-580 (2007). doi:10.1128/JCM.02262-06

23. V. Martella, K. Bányai, M. Ciarlet, M. Iturriza-Gómara, E. Lorusso, S. De Grazia et al., Virology 344, 509-519 (2006). doi: 10.1016/j.virol.2005.08.029

24. J. Matthijnssens, M. Rahman, X. Yang, T. Delbeke, I. Arijs, J. Kabue et al., J. Clin. Microbiol. 44, 1801-1809 (2006). doi: 10.1128/JCM.44.5.1801-1809.2006 
25. J.R. Gentsch, R. Glass, P. Woods, V. Gouvea, M. Gorziglia, J. Flores et al., J. Clin. Microbiol. 30, 1365-1373 (1992)

26. V. Gouvea, R. Glass, P. Woods, K. Taniguchi, H.F. Clark, B. Forrester et al., J. Clin. Microbiol. 28, 276-282 (1990)

27. C. Lee, Y. Wang, C. Kao, C. Zao, C.Y. Lee, H. Chen, J. Clin. Microbiol. 38, 4471-4477 (2000)

28. S. Altschul, T. Madden, A. Schäffer, J. Zhang, Z. Zhang, W. Miller et al., Nucleic Acids Res. 25, 3389-3402 (1997). doi: 10.1093/nar/25.17.3389

29. S. Kumar, K. Tamura, I. Jakobsen, M. Nei, Bioinformatics 17, 1244-1245 (2001). doi:10.1093/bioinformatics/17.12.1244

30. M. Ciarlet, M.K. Estes, M.E. Conner, Arch. Virol. 142, 10591069 (1997). doi:10.1007/s007050050142

31. C. Arias, P. Romero, V. Alvarez, S. López, J. Virol. 70, 58325839 (1996)

32. M. Gorziglia, G. Larralde, A. Kapikian, R. Chanock, Proc. Natl. Acad. Sci. U.S.A. 87, 7155-7159 (1990). doi:10.1073/pnas.87. 18.7155

33. F. Liprandi, M. Gerder, Z. Bastidas, J. López, F. Pujol, J. Ludert et al., Virology 315, 373-380 (2003). doi:10.1016/S0042-6822(03) 00534-8

34. K. Chang, A. Parwani, L.J. Saif, Arch. Virol. 140, 1279-1283 (1995). doi:10.1007/BF01322753

35. N. Cunliffe, J.R. Gentsch, C. Kirkwood, J. Gondwe, W. Dove, O. Nakagomi et al., Virology 274, 309-320 (2000). doi:10.1006/ viro. 2000.0456

36. E. Palombo, R. Clark, R. Bishop, J. Med. Virol. 60, 56-62 (2000). doi $: 10.1002 /($ SICI)1096-9071(200001)60:1<56::AID-JMV10> 3.0.CO;2-7

37. V. Parreño, K. Bok, F. Fernández, J. Gómez, Arch. Virol. 149, 2465-2471 (2004). doi:10.1007/s00705-004-0371-2

38. K. Fukai, K. Yamada, K. Inoue, Virus Res. 114, 167-171 (2005). doi:10.1016/j.virusres.2005.05.009

39. K. Fukai, T. Takahashi, K. Tajima, S. Koike, K. Iwane, K. Inoue, Vet. Microbiol. 123, 217-224 (2007)

40. M. Adah, A. Wade, K. Taniguchi, J. Clin. Microbiol. 39, 39693975 (2001). doi:10.1128/JCM.39.11.3969-3975.2001

41. M. Adah, S. Nagashima, M. Wakuda, K. Taniguchi, J. Clin. Microbiol. 41, 3945-3950 (2003). doi:10.1128/JCM.41.8.39453950.2003
42. M. Cooney, R. Gorrell, E. Palombo, J. Med. Microbiol. 50, 462-467 (2001)

43. J. Holmes, C. Kirkwood, G. Gerna, J. Clemens, M. Rao, A. Naficy et al., Arch. Virol. 144, 1381-1396 (1999). doi: 10.1007/s007050050594

44. M. Ciarlet, Y. Hoshino, F. Liprandi, J. Virol. 71, 8213-8220 (1997)

45. H.F. Clark, P.A. Offit, R. Glass, R.L. Ward, in Vaccines, 4th edn., ed. by S.A. Plotkin, W.A. Orenstein (Elsevier, Inc., Philadelphia, PA, 2004), pp. 1327-1345

46. D.R. Snodgrass, T. Fitzgerald, I. Campbell, F. Scott, G. Browning, D. Miller et al., J. Clin. Microbiol. 28, 504-507 (1990)

47. M. Ciarlet, F. Reggeti, C.I. Piña, F. Liprandi, J. Clin. Microbiol. 32, 2609-2612 (1994)

48. M. Ciarlet, J.E. Ludert, F. Liprandi, Arch. Virol. 140, 437-451 (1995). doi:10.1007/BF01718422

49. P. Iša, A. Wood, T. Netherwood, M. Ciarlet, H. Imagawa, D.R. Snodgrass, Arch. Virol. 141, 1601-1612 (1996). doi:10.1007/ BF01718285

50. S. Matsuno, A. Hasegawa, A. Mukoyama, S. Inouye, J. Virol. 54, 623-624 (1985)

51. A. Gerna, L. Sarasini, L. Zentilin, A. Di Matteo, P. Miranda, M. Parea et al., Arch. Virol. 112, 27-40 (1990). doi:10.1007/ BF01348983

52. K. Bányai, J.R. Gentsch, R. Glass, G. Szucs, Epidemiol. Infect. 130, 107-112 (2003). doi:10.1017/S0950268802007975

53. J. Matthijnssens, M. Rahman, M. Ciarlet, M. Van Ranst, in Viruses in the Environment, ed. by E. Palombo (Research Signpost, Trivandrum, India, 2008), pp. 171-318

54. Y. Hoshino, S. Honma, R. Jones, N. Santos, O. Nakagomi, T. Nakagomi et al., Virology 345, 1-12 (2006). doi:10.1016/j.virol. 2005.09.003

55. G. Gerna, J. Sears, Y. Hoshino, A.D. Steele, O. Nakagomi, A. Sarasini et al., Virology 200, 66-71 (1994). doi:10.1006/viro. 1994.1163

56. V. Martella, M. Ciarlet, A. Lavazza, A. Camarda, E. Lorusso, V. Terio, D. Ricci, F. Cariola, M. Gentile, A. Cavalli, M. Camero, N. Decaro, C. Buonavoglia, Vet. Microbiol. 111, 117-124 (2005). doi:10.1016/j.vetmic.2005.10.005 